\title{
CHILE Y COREA DEL SUR: 1973 Y LA CONFIGURACIÓN DE UNA ALIANZA ESTRATÉGICA*
}

\author{
CHILE AND SOUTH KOREA: 1973 AND THE CONFIGURATION \\ OF A STRATEGIC ALLIANCE
}

\author{
César Ross**
}

\begin{abstract}
Situado en contexto de la tensión entre el aislamiento internacional y la supervivencia del gobierno cívico militar de Chile, este trabajo se concentra en examinar el primer momento (1973-1975) de lo que podría llamarse una relación aparentemente improbable. La hipótesis de este trabajo respecto de este primer momento (post-1973), plantea que la consecuencia principal fue crear las condiciones para que los gobiernos encabezados por Park y Pinochet se reconociesen como pares dentro de un contexto adverso (aislamiento y resistencia interna real o virtual), de modo de configurar las relaciones bilaterales con sentido estratégico, dentro de una coyuntura crítica de uno de los líderes (atentado), que potencialmente podría ocurrir a su homónimo en cualquier momento, lo que operó como catalizador de un vínculo que se configuró rápidamente con las características de lo que podría denominarse como una alianza estratégica.

La clave de este primer momento fue enfatizar la supervivencia de ambos regímenes, mediante un vínculo que involucró a ambos líderes de manera política y personal, en un capítulo hasta ahora desconocido de la historia internacional del régimen cívico militar de Chile.
\end{abstract}

Palabras claves: Chile, Corea del Sur, política exterior, quiebre institucional, régimen militar.

Set in the context of the tension between international isolation and Chile's civil-military government's survival, this paper concentrates on examining the first moment (1973-1975) of what could be called an unlikely relationship.

This work's hypothesis regarding this first moment (post-1973) states that the main consequence was to create the conditions for the governments headed by Park and Pinochet to recognize themselves as peers within an adverse context (isolation and real or virtual internal resistance ). To configure bilateral relations with a strategic sense, within a critical juncture of one of the leaders (attack), which could potentially happen to his homonym at any time, acting as a catalyst for a relationship that was quickly configured with the characteristics of what could be called a strategic alliance.

The key to this first moment was to emphasize the survival of both regimes, through a bond that involved both leaders politically and personally, in a hitherto unknown chapter in the international history of Chile's civil-military regime.

Key words: Chile, South Korea, foreign policy, institutional breakdown, military regime.

\section{Introducción}

Desde el punto de vista teórico, y coincidiendo con la idea que de que las fronteras entre historia diplomática, historia internacional, historia mundial/ global e historia transnacional son relativamente tenues (Williams, 2012), este trabajo procura articular tres enfoques específicos cuyas fronteras también son tenues: historia del tiempo presente (HTP), relaciones internacionales (RRII) e historia de las relaciones internacionales (HRRII). Se busca problematizar (Hollis-Smith, 1990), desde un punto de vista histórico (Braudel, 1968), las claves de la relación entre estos dos países, entre 1973 y
1989, bajo una perspectiva integradora (Huguet, 2001). En esta opción intentamos articular, tanto un enfoque del tipo top down, que nos provee las RRII y las HRRII; con un enfoque de tipo bottom up, que buscamos desarrollar desde los métodos de Historia, que nos ofrece la HTP, contenida en archivos y en la historiografía.

Las relaciones de Chile con el Este de Asia, especialmente durante los años de la Guerra Fría, están dentro de lo que Peter Smith habría catalogado como de alianza improbable (Smith, 2003). Sin embargo, a nuestro juicio dicha aseveración está errada o al menos incompleta: entre estas dos orillas del Pacífico sí hubo alianzas estratégicas

\footnotetext{
* This work was supported by the Seed Program for Korean Studies through the Ministry of Education of the Republic of Korea and the Korean Studies Promotion Service (KSPS) of the Academy of Korean Studies (AKS-2014-INC-2230007). Además, este trabajo es un resultado parcial del proyecto Fondecyt Regular No 1200168.

** Universidad de Santiago de Chile. Santiago, Chile. Correo electrónico: cesar.ross@usach.cl
} 
robustas, una de estas fue entre Chile y Corea del Sur, una historia tan fascinante como desconocida, que se inserta en una ya no tan nueva lectura de las relaciones del régimen cívico-militar de Chile y el Este de Asia (Ross, 2007). Este vínculo, junto con el de Japón y China, constituyó una pieza clave de la trama básica con la que Chile consolidó su modelo de relaciones con el Este de Asia, cuyos pilares fueron el pragmatismo y las relaciones económicas. A este respecto existe una no tan extensa bibliografía donde los trabajos generales de autores como Joaquín Fermandois y Heraldo Muñoz, han hecho una contribución importante.

A comienzos de la década de 1970 las relaciones entre Chile y Corea del Sur estaban en el campo de lo improbable, sin embargo, este vínculo se desarrolló a un nivel muy alto y en un plazo muy corto.

La singularidad de este vínculo no solo responde a lo que llamaremos la excepcionalidad asiática ${ }^{1}$ para la política internacional de Chile de esta época, sino que a la proximidad estructural entre los regímenes de ambos países bajo los mandatos de Park Chunghee (1963-1979) y Augusto Pinochet (1973-1988).

El momento de inflexión en la tendencia precedente fue el golpe de Estado del 11 de septiembre de 1973 en Chile. A partir de ese momento el vínculo entre sus líderes se comenzó a estrechar y los destinos de los países a converger. De este modo, lo improbable se hizo posible y una heterodoxa relación sur-sur tendió a desarrollarse, más allá del ámbito público, en tanto los pasajes más elocuentes de esta relación ocurrieron en el secretismo de la diplomacia dictatorial de los primeros años del gobierno cívico-militar encabezado por Pinochet. Esto en un contexto extremadamente frágil para el gobierno de Park y para el propio régimen chileno que, en una secuencia de ensayos y errores, aprendían cómo identificar países no hostiles a su posición y establecer con ellos sus puntos de apoyo en un escenario que era de suyo muy adverso.

En lo principal, en este trabajo preguntamos cuál fue la consecuencia principal del quiebre de la democracia en Chile en las relaciones con Corea del Sur.

Mi respuesta, o hipótesis, plantea que la principal consecuencia fue crear las condiciones para que los gobiernos encabezados por Park y Pinochet se reconociesen como pares dentro de un contexto adverso (aislamiento y resistencia interna real o virtual), de modo de configurar las relaciones bilaterales con sentido estratégico, dentro de una coyuntura crítica para el líder coreano (1974, atentado), que potencialmente podría ocurrir a su homónimo chileno en cualquier momento, lo que operó como catalizador de un vínculo que se configuró rápidamente con las características de lo que podría denominarse una alianza estratégica.

El objetivo central de este trabajo es identificar la consecuencia principal que el golpe de Estado del 11 de septiembre en Chile tuvo en las relaciones entre Chile y Corea del Sur. Este objetivo se inscribe dentro de una línea de investigación, donde se ha estudiado el rol de los países del Este y Noreste de Asia en la mitigación del aislamiento internacional del régimen cívico militar de Chile (Ross, 2007) (2014).

Desde el punto de vista de las fuentes, se ha utilizado bibliografía que sostiene teórica y empíricamente el razonamiento general desplegado en la argumentación del trabajo, datos de fuentes seriadas y documentación proveniente del Archivo General Histórico del Ministerio de Relaciones Exteriores de Chile (AGHMRREE).

El artículo está organizado en cuatro partes. La primera, referida a lo que he denominado la excepcionalidad asiática frente a Chile. La segunda, al establecimiento de las relaciones diplomáticas. $\mathrm{La}$ tercera, titulada el nuevo estatus de las relaciones bilaterales. La cuarta, denominada, Chile y Corea, la configuración de un vínculo estratégico.

\section{El gobierno chileno (1973-1989) y la excepcionalidad asiática}

Pese al aislamiento político de la dictadura de Pinochet, esta logro sobrevivir 17 años en medio de un fuerte asedio internacional de diversos gobiernos que habían mantenido una buena relación con el gobierno de Allende (como Francia, Italia y la URSS, entre otros), de partidos políticos internacionales (PC, DC, PS, Social Democracia, etc.), de ONG, de DD.HH., de redes de intelectuales y de artistas organizados en torno a Human Rights Watch, de múltiples organizaciones opuestas a las dictaduras del mundo, de parte del Congreso de Estados Unidos y, en algún momento, de los propios gobiernos de Estados Unidos (Spring, 1980) (Sater, Morley, y McGillion, 2015).

Según Fermandois: "El Estado chileno pudo probarse en una situación de extremo aislamiento y amenaza, en donde su política exterior [fue] una combinación de acierto y azar" (1991, p. 445). En 
un contexto en el que la oposición interna se fue incrementando dinámicamente, sobre todo en la década de los años 80 donde la paradoja general tensionó dos fenómenos aparentemente contradictorios: la resistencia interna y externa al gobierno cívico militar encabezado por Pinochet; y, por otra, los 17 años que logró sobrevivir este gobierno.

En el plano interno, podría plantearse que sobrevivió por cuatro razones principales: 1) el terror impuesto, lo que inhibió acciones colectivas por casi una década; 2) la eficiencia de la policía política para neutralizar a la oposición; 3) la capacidad de Pinochet para concentrar el poder y mantener la lealtad de sus subordinados; y 4) el apoyo irrestricto de una clase empresarial (tradicional y emergente) que participó de los beneficios económicos del desmantelamiento de las empresas y recursos del Estado.

En el ámbito internacional, la dictadura sobrevivió por una política exterior bastante más sofisticada que la representada por el llamado "estilo diplomático pretoriano ideológico", cuya acción tuvo especial éxito con países de regímenes políticos del mismo signo y con países como los asiáticos, cuyas economías despegaban tímidamente en 1973 (con la excepción de Japón que ya era una potencia), pero que alcanzaron un estatus clave en los años siguientes ${ }^{2}$.

La reacción de los países del Este asiático hacia Chile, después del quiebre de la democracia del 11 de septiembre de 1973, fue favorable, toda vez que su indiferencia a la situación política chilena no se sumaba negativamente al repudio que el nuevo régimen recibió. De allí, la política del gobierno militar hacia estos países fue de la mayor colaboración posible, con vistas a combatir el aislamiento en el que se encontraba. El gobierno aprendió rápidamente que sus relaciones con el Este asiático debían ser pragmáticas y que los agentes de estas debían ser los diplomáticos, los tecnócratas recién incorporados a la alta burocracia estatal y los hombres de negocios (Ross, 2007) (Toro-Ibacache, 2018).

Con China, la relación estuvo acotada a los intereses económicos bilaterales y a una cierta convergencia de enfoque acerca de las reformas que ambas economías requerían en los 70 (Sigmund, 1993), complementada con una diplomacia funcional y relativamente fría $^{3}$, entre otras cosas por la cercanía chilena con el régimen de Taiwán (Álvarez, 2017).

Con Japón, la relación estuvo centrada en lo económico, con un componente político funcional (relaciones transaccionales) y con un mayor apoyo político japonés a la supervivencia política del gobierno militar, todo ello se explica por la necesidad que tenía de los recursos naturales de Chile ${ }^{4}$.

Con Hong Kong, aún en calidad de territorio británico de ultramar, le unía una relación muy limitada a lo económico, donde las dimensiones políticas y diplomáticas estaban relacionadas al gobierno de Londres.

Con Taiwán o Taipei, la relación estuvo centrada en lo económico, con una proximidad sistémica (antimarxismo, autoritarismo político y liberalismo económico) que se extendió de manera importante a los temas de seguridad, cuyos detalles están por ser investigados con mayor detenimiento en el futuro. Esta relación estaba condicionada por el factor "China", por cuanto el estatus de relaciones diplomáticas de Chile con esta impedía el desarrollo del vínculo Chile-Taiwán.

Con Corea del Sur, al igual que el caso de Taiwán, se trató de una relación centrada crecientemente en lo económico, con una proximidad sistémica (antimarxismo, autoritarismo político y liberalismo económico), pero con la ausencia de un factor disruptivo crítico, porque en este caso la cercanía de ambos gobiernos con Estados Unidos, facilitaba las relaciones de Chile con ambos (Ross y Álvarez, 2018).

\section{Establecimiento de las relaciones diplomáticas}

Las relaciones políticas entre Chile y Corea del Sur se iniciaron en 1962, bajo las rigideces propias de la Guerra Fría, razón por la que Chile se vio forzado a tomar partido en la tensión entre Corea del Sur y Corea del Norte. Como se ha planteado en el libro conmemorativo editado por la Biblioteca del Congreso Nacional, "Los roces entre ambas naciones llegaron a la Asamblea de Naciones Unidas" (2012, p. 39), donde los surcoreanos lograron el apoyo para imponer su voluntad para que la ONU mediara en el proceso de reunificación. Chile apoyó dicha moción, lo que estrechó el vínculo político entre ambos Estados.

Sin embargo, aún se trataba de una relación de baja intensidad. Recién después de 6 años de iniciadas estas relaciones se suscribió un convenio básico de cooperación económica y técnica, que incluía desarrollo de proyectos en Chile y capacitación de chilenos en Corea del Sur. Pese a esto, el ritmo e 
intensidad de las relaciones se mantuvieron en un nivel muy bajo.

En 1970, el triunfo electoral de la Unidad Popular, encabezada por Salvador Allende, implicó un cambio importante en las relaciones internacionales de Chile. Sin romper relaciones con los países alineados con Estados Unidos, se abrieron relaciones con los países de la órbita socialista (Fermandois, 1985). Allende asumió el poder el 4 de noviembre de 1970 y el día 16 del mismo mes se establecieron las relaciones con Corea del Norte.

Aparentemente, no hubo más actividad en el vínculo entre Chile y Corea del Norte, exceptuado por un viaje del canciller chileno, Clodomiro Almeyda, que en gira por Asia visitó ese país, instancia "donde las autoridades locales le plantearon su interés en comprar cobre chileno" (BCN, 2012, p. 39) (no hay registro de algún intercambio). A mediados de 1973 se suscribió un convenio de intercambio cultural en momentos que el gobierno de Allende estaba llegando a su fin y a partir del 12 de septiembre de 1973, las prioridades del nuevo gobierno fueron en la dirección opuesta, por lo que ninguna de las pocas iniciativas impulsadas en esos años llegó a prosperar.

Respecto de Corea del Sur, y basados en los datos recopilados por el Banco Central de Chile, en sus "indicadores sociales y económicos" (Tabla 1), tendríamos que colegir que el comercio bilateral fue inexistente, en tanto aparece en cero para los años 1970 a 1973, incluso hasta 1976.
Tabla 1. Chile 1973-1976: Comercio con Corea del Sur. (En millones de US\$)

\begin{tabular}{ccccc}
\hline Año & Importaciones & Exportaciones & Saldo & Total \\
\hline 1973 & 0,00 & 0,00 & 0,00 & 0,00 \\
1974 & 0,00 & 0,00 & 0,00 & 0,00 \\
1975 & 0,00 & 0,00 & 0,00 & 0,00 \\
1976 & 0,00 & 3,50 & 3,50 & 3,50 \\
\hline
\end{tabular}

Fuente: Elaboración propia, sobre la base de indicadores sociales y económicos del Banco Central.

Sin embargo, la información recogida por la representación diplomática chilena acreditada en Seúl (Tabla 2) y registrada en el Archivo Histórico del Ministerio de Relaciones Exteriores de Chile, revela que sí hubo intercambio comercial con Corea del Sur en este mismo período, aunque en un volumen muy reducido.

Por pequeños que fueran los montos de este incipiente comercio, se advierte la presencia de su estructura clásica de un intercambio complementario, entre un productor de bienes manufacturados como Corea del Sur, y un productor de recursos naturales como Chile. En una progresión crecientemente compleja, esta misma estructura se mantuvo inalterada en los años y décadas siguientes.

En síntesis, y utilizando la metáfora del comienzo de este trabajo, podría afirmarse que, en estos años, estos vínculos económicos, que he clasificado como improbables, se mantuvieron en ese mismo

Tabla 2. Chile 1971-1974: Comercio con Corea del Sur. (En US\$ FOB)

\begin{tabular}{lrrrr}
\hline \multicolumn{1}{c}{ Exportaciones } & 1971 & 1972 & 1973 & $1974(1)$ \\
\hline Harina de pescado & 1.981 .000 & 562.994 & No hubo & 0 \\
Cobre electrolítico & 536.435 & 0 & No hubo & 0 \\
Cobre, plata y minerales & 0 & 2.173 .410 & No hubo & 0 \\
Cobre cemantación (sic) & 0 & 0 & 0 & $8.614(2)$ \\
\multicolumn{1}{c}{ Importaciones } & 1971 & 1972 & 1973 & $1974(3)$ \\
\hline Suntuarios & 3.523 & 0 & 0 & 0 \\
Artículos varios & 0 & 839.211 & 378.410 & 0 \\
Artículos para calzados & 0 & 0 & 0 & 544 \\
Pilas eléctricas & 0 & 0 & 0 & 891 \\
\hline
\end{tabular}

Fuente: Elaboración propia, sobre la base de información contenida en el AHMRREE, Oficio Ordinario, 1975, № 7.

Notas:

(1) Hasta el mes de septiembre.

(2) Cifra incompleta. En el original aparece como "8.614._ _, lo que no permite saber si faltan o no algunos números. Indica que se trata de 9.500 toneladas.

(3) Hasta el mes de septiembre. 
estado, pero las relaciones políticas alcanzaron un estatus más alto.

\section{El nuevo estatus de las relaciones bilaterales}

\subsection{Reconocimiento del gobierno cívico militar de Chile}

En respuesta a la nota del 12 de septiembre de 1973, que el nuevo gobierno de Chile envió a los demás gobiernos con los países con que se tenía relación, Seúl respondió con un documento firmado el 24 de septiembre de 1973 dando formal reconocimiento al nuevo gobierno de Chile. En esta nota, además, se leía "Es nuestro sincero deseo que la cordial amistad y cooperación entre la República de Corea y la República de Chile sea fomentada y fortalecida en el futuro" (AGHMRREE, 1973).

Un mes después del reconocimiento formal, el 23 de octubre, volvió a Santiago el embajador residente en Corea del Sur en Chile, Choon Hee Kang, para reasumir sus funciones y restablecer la rutina de las relaciones diplomáticas.

En noviembre de 1973, la Embajada de Corea en Santiago le informó en detalle al Ministerio de Relaciones Exteriores de Chile acerca del total de sus funcionarios, remitiéndole una lista con nombres y cargos, que implicaba a cinco funcionarios, incluidos el embajador, el ministro consejero, el tercer secretario, el agregado y un teniente coronel, que cumplía la función de agregado militar, naval y aéreo.

En diciembre de 1973, los presidentes Park y Pinochet intercambiaron tarjetas de Navidad, por medio de la comunicación diplomática, comunicaciones en las que, como de rutina en el uso protocolar, aprovecharon la oportunidad para "reiterarse la seguridad de su más alta y distinguida consideración" (AGHMRREEb, 1975).

Se acabó 1973 y parecía que nada había ocurrido en estas relaciones o que pese a todo lo ocurrido, el vínculo se mantenía y, como veremos, se ampliaría y profundizaría.

Durante 1974 solo se registró la visita a Santiago de Chu Cheng Yoon, ministro de Cultura e Informaciones de Corea del Sur. Sin embargo, fue más que una visita técnica, ya que se reunió con los integrantes de la junta de gobierno de Chile. $\mathrm{Al}$ año siguiente Chile abrió su embajada en Seúl.

\subsection{Instalación de Embajada de Chile en Seúl}

Con motivo de la apertura de la Embajada de Chile en Seúl, se elaboró un Informe (AGHMRREEb, 1975) acerca de la instalación y las primeras impresiones que el país produjo en el personal diplomático chileno, aunque como el extenso documento fue suscrito por el entonces encargado de negocios, Renán Sánchez Vargas, la mayor parte de los juicios debería ser atribuibles a él mismo.

El Informe no solo revela la acuciosidad que un documento como este demanda, lo que nos ayuda a conocer aspectos objetivos de Corea y de la propia instalación de la Embajada, sino que a comprender la subjetividad que podría estar involucrada en este vínculo.

El encargado de negocios aludido llegó a Seúl alrededor del 5 de marzo de 1975 . Se trata de un mes en donde las temperaturas máximas rodean los $10^{\circ} \mathrm{C}$ y las mínimas los $-5^{\circ}$, todo esto da una bienvenida relativamente hostil. A ello se agregó el problema de los escasos recursos financieros otorgados para la apertura y a que en Corea debía pagarse 6 meses de arriendo por anticipado, lo que consumió el total de los recursos dispuestos para la instalación, ascendentes a US\$ 15000.00. Pese a estas dificultades iniciales, ya el día 7 de abril, el encargado de negocios y el agregado militar entraron en funciones.

El encargado de negocios, por ejemplo, informó con incertidumbre por el desempeño del trabajo, de la situación de Corea del Sur y la compleja relación con los coreanos. Respecto de lo primero, señalaba que:

Las comunicaciones urgentes sufren atrasos o interferencias que impiden una labor efectiva, especialmente en momentos en que el sudeste asiático [Guerra de Vietnam] presenta situaciones críticas y Corea del Sur puede vivir momentos difíciles de predecir (AGHMRREEb, 1975).

Esta percepción se vio reforzada, por ejemplo, con motivo de la visita de la esposa del Embajador Fontaine, a la esposa del ministro de Relaciones Exteriores de Corea, Dong-jo Kim, la que debió ser suspendida por una alarma antiaérea (AGHMRREEe, 1975), lo que resultó ser una forma común de entrenamiento para la población local, precisamente por que dicho peligro estaba dentro de lo previsible. 
En cuanto a lo segundo, la situación era mucho más difícil respecto del país y su población. El propio embajador, en oficio remitido al canciller chileno, se lamentaba de que en Corea faltaba:

Acceso a diversos elementos de uso normal en el mundo occidental, pero que acá no se usan ni se pueden obtener (lacre, desodorante, remedios especiales, etc. por nombrar más que algunos) con relativa facilidad y a menudo con ninguna facilidad" (AGHMRREEc, 1975).

Por su parte, el encargado de negocios revela en sus palabras un desembozado prejuicio cultural. No solo refiere a Corea como "un país donde el inglés se habla pésimo y se entiende peor" (AGHMRREEb, 1975), sino que se refiere a los coreanos con displicencia, argumentando por una superioridad nacional, pese a que su comentario lo desprende de un reiterado halago de los coreanos hacia Chile:

Sin ninguna excepción, todas las entrevistas fueron casi calcadas...expresaron su simpatía por el Gobierno de S.E. el General Augusto Pinochet Ugarte y, además, mencionaron el hecho de que tanto el pueblo coreano como el chileno tenían mucho en común y eran 'lo únicos que habían sido capaces de derrotar al comunismo' (AGHMRREEb, 1975).

Agregaba Sánchez, "pongo dicha frase, expresada por el Sr. Viceministro, entre comillas, ya que en la comparación no se hace justicia al pueblo chileno, por razones obvias. Además, he podido observar que somos superiores" (AGHMRREEb, 1975).

En esta misma línea de ideas, en otra parte del informe, Sánchez se refiere a la cultura de negociación de los coreanos, afirmando que "no creo se comprometan a dar nada que no les reporte por lo menos el doble de utilidad, ya sea en lo político, en lo económico o en lo social" (AGHMRREEb, 1975). Para dar sustento a sus juicios, el encargado de negocios sostenía que en el mes de residencia había recorrido la ciudad entera, que había tratado con altos funcionarios y con gente del pueblo, por lo que había logrado "captar esta forma de ser de los coreanos, que tampoco demuestran ser muy despiertos, ni dúctiles" (AGHMRREEb, 1975).
Para cerrar el punto agregaba, "en resumen, todo está organizado para sacarle plata y provecho al extranjero, pero no darle nada o casi nada a cambio" (AGHMRREEb, 1975).

Al final del Informe Sánchez agrega un dato que completa de ilustrar la atmósfera que se vivía en Corea del Sur de 1975:

Los guardias policiales de la entrada al Ministerio de Relaciones Exteriores son bastante descomedidos e insolentes, no solo con el suscrito, sino con el Cuerpo Diplomático en general, pues me he impuesto por los Embajadores que constantemente están presentándose problemas de esta índole e incluso se han acentuado después del atentado que costó la vida a la señora del Presidente Park (AGHMRREEb, 1975).

A todo lo anterior se agregó la sospecha, aparentemente fundada, de que la correspondencia de la Embajada de Chile era abierta antes de llegar a la sede chilena. Según oficio enviado por el embajador Leopoldo Fontaine al ministro de Relaciones Exteriores, "la correspondencia recibida y enviada por correo certificado o normal sufre atrasos de más de 20 días y que, finalmente, dicha correspondencia es regularmente sometida a "inspección"" (AGHMRREEc, 1975).

\subsection{El nuevo estatus de las relaciones bilaterales}

A partir del 11 de septiembre de 1973, las relaciones bilaterales se elevaron de estatus, por la coincidencia ideológica de los regímenes, por la inesperada cercanía de Park con Pinochet y porque Chile abrió una embajada residente en Seúl, con el efecto que ello podría conllevar en la interacción diplomática.

Pese a la baja interacción bilateral precedente, la Cancillería chilena tenía una perspectiva muy precisa respecto de su organización político-administrativa, del proceso de transformación económica de Corea del Sur y de su política exterior, cuya descripción tiene total vigencia:

El gobierno coreano contempla una vigorosa política exterior. Esta política está diseñada para reforzar la seguridad del país frente a Corea del Norte, para buscar la unificación 
del país bajo auspicio de naciones Unidas y para contrarrestar los esfuerzos diplomáticos norcoreanos destinados a frustrar dichos objetivos (AGHMRREEa, 1975).

Una vez establecido en Seúl, y con sus credenciales presentadas el 14 de mayo de 1975, el embajador Leopoldo Fontaine hizo las visitas protocolares de rigor, que, por su propia naturaleza, permitieron situar a los actores cara a cara y crear oportunidades para elevar las relaciones a un nivel muy superior 5 .

Se le informó que en la tarde del mismo día 14 de mayo lo recibiría el primer ministro, Sr. Kim Jong-Pil ${ }^{6}$ (yerno del presidente Park $^{7}$ ), y que el día 16 del mismo mes le recibirían el viceministro de Relaciones Exteriores, el jefe de los asuntos americanos del Ministerio de Relaciones Exteriores y el presidente de la Corte Suprema, alcalde de Seúl, al ministro de Defensa y las visitas al cuerpo diplomático.

Según el Informe de Fontaine (AGHMRREEe, 1975), la visita al primer ministro, Sr. Kim Jong-Pil, se refirió a temas generales, pero fue muy reveladora la solicitud que, si la Embajada de Chile tenía algún problema, debían contactarlo directamente a él, lo que difiere del curso regular de estas relaciones, que usualmente deben ser canalizadas por medio del Ministerio de Relaciones Exteriores de Chile. No se sabe si ello se debió a que en pocos meses el titular de dicha cartera (Dong-jo Kim) dejaría su cargo (18 de diciembre de 1975), o si se debía a que con Chile se buscaba tener un trato preferente, debido al vínculo que se había formado entre Park y Pinochet, desde agosto de 1974.

En la visita al viceministro de Relaciones Exteriores, el embajador Fontaine informó acerca de lo que él expuso, pero no de la reacción de la autoridad coreana. Según su informe, en esta cita habló del cambio de gobierno, de la propaganda externa contra Chile, acerca de las condiciones económicas en que se recibió al país y la relación con sus vecinos ${ }^{8}$.

La cita con el presidente de la Corte Suprema estuvo delineada por las interrogantes de la autoridad coreana. Básicamente, le interesaba saber acerca de la justicia en Chile antes y después del 11 de septiembre de 1973. El embajador le dio la explicación estándar respecto de la división de poderes y que la Corte Suprema de Chile había reconocido la "situación" (sic) al nuevo gobierno y desde entonces tenía absoluta independencia (AGHMRREEe, 1975). El informe no entrega datos acerca de la reacción del presidente de la Corte Suprema.

En la reunión con el alcalde de Seúl, definida como muy cordial, como todas las sostenidas con las autoridades coreanas, solo se tocaron temas formales de la presencia de una nueva embajada en la ciudad.

La reunión con el ministro de Defensa fue más importante, por cuanto la Esmeralda, busque escuela de Chile, visitaría Corea del Sur y el embajador debía intentar comprometer la presencia del ministro para la ocasión. El jefe de la defensa coreana respondió con una cortesía, afirmando que lo haría con el mayor agrado, pero que no podía asegurarlo, debido a la situación de tensión que vivían. El embajador Fontaine no explicó a qué se refería con dicha situación.

En este período de instalación, el embajador Fontaine informó acerca de todas las reuniones que tuvo con el cuerpo diplomático residente, donde se reflejó la división internacional en torno a la dictadura chilena. Por una parte, Argentina, Brasil y Uruguay que coincidían con Chile ${ }^{9}$. Por otra, Perú e Italia, que eran abiertamente opuestos a Chile ${ }^{10}$. Finalmente, Suiza que era tradicionalmente neutral.

Este nuevo estatus, además, se condijo con la planificación del viaje a Corea del Sur del almirante José Toribio Merino Castro, información registrada en una comunicación del 5 de noviembre de 1975, del ministro de Relaciones Exteriores de Chile, Patricio Carvajal Prado, al embajador de Chile en Seúl, Leopoldo Fontaine, y suscrita por Jorge Berguño Barnes, en calidad de director de Relaciones Internacionales (AGHMRREEf, 1975).

En síntesis, entre el 24 de septiembre de 1973, cuando el gobierno coreano reconoció formalmente al nuevo gobierno de Chile y junio de 1975, cuando el embajador chileno concluyó las visitas protocolares, sellaron el nuevo estatus de la Embajada residente de Chile en Seúl y la relación bilateral quedó situada en una posición, que bien puede clasificarse como alianza estratégica.

\section{Chile y Corea: la configuración de una alianza estratégica}

La visión clásica de la Guerra Fría tiende a esquematizar la organización de los países en función de un mundo bipolar (Spykman, 1942) (Morgenthau, 1948), planteando una correlación muy alta entre 
quienes formaban parte de un bloque y el líder del mismo. Sin embargo, la alineación diversa de los actores demostró que no se podía inferir acerca de las relaciones internacionales solo a partir de la existencia de dos bloques y evidenció que la conflictividad de la Guerra Fría tenía una escala geográfica mayor, por lo que la misma expresión que la denominaba resultaba insuficiente.

En este contexto, Arne Westad (2006) acuñó la categoría de "Guerra Fría Global", para denotar que este fenómeno también tuvo su correlato en países o Estados no centrales, donde la polarización política e ideológica se desarrolló activamente: No solo los alineó con los líderes e integrantes de uno y otro bando, sino que impactó en las conflictividades políticas locales. Así, la política exterior y las relaciones internacionales de cada Estado se configuraron en una trama compleja, donde los vínculos se desarrollaron tanto con los aliados del líder como con otros cuya alineación podía ser ideológicamente opuesta y que derivara en alianzas estratégicas improbables ${ }^{11}$.

\subsection{El azar: Chile y Corea}

La singularidad de este caso de relaciones bilaterales radica en que, a diferencia de otros regímenes dictatoriales también considerados "parias", Chile y Corea del Sur practicaron una política antimarxista acorde a la política de Estados Unidos (sobre todo de Richard Nixon, Gerald Ford y Ronald Reagan) e Inglaterra (de Margaret Thatcher) y desarrollaron una economía abierta que convencía a dichas potencias, sobre todo en la década de 1980, período de implementación de las políticas económicas neoliberales, de las que Reagan y Thatcher fueron líderes.

Ambos regímenes combinaron un autoritarismo político que no solo se desplegó hacia sus adversarios, sino que hacia los empresarios que eventualmente se opusieran a sus políticas económicas (Rodrik, 1993). En este contexto, dichas medidas se implementaron con suficiente estabilidad de reglas como para ofrecer resultados positivos en el mediano y largo plazo.

La importancia local de este vínculo bilateral fue enfatizar un modelo de relaciones internacionales pragmáticas, disociado de consideraciones políticas ${ }^{12}$, así como reforzar su carácter instrumental y consolidar el enfoque neorrealista y neoliberal de la política exterior de Chile. Estos rasgos, presentes antes de 1973 (Ross, 2014), fueron profundizados en este período histórico y preservados como la base de la política exterior de Chile, desde entonces y hasta el presente ${ }^{13}$.

Sin perjuicio de la denominación que se elija, en ambos casos prevaleció el establecimiento de regímenes autoritarios que afirmaron su poder y pervivencia en la represión a sus opositores y en el éxito económico (Domínguez, 2011), piezas claves y encadenadas en la concepción y en el desarrollo de ambos modelos (Ross, 2016).

Para denominar estos regímenes, la bibliografía especializada ha utilizado varias categorías de clasificación sin que se haya hecho mayor debate acerca de las mismas.

En el caso coreano (García-Blanch, 2002), se impulsó el llamado "régimen de industrialización burocrático-autoritario" (Romero, 2005), basado en un modelo compuesto por tres actores clave: los militares, los empresarios y la burocracia (León, 2006). Su característica fue el autoritarismo político, sistema sustentado en la Constitución Yushin (1972) de tipo dictatorial, y su esfuerzo por la disolución de la Asamblea Nacional, en la concentración del poder y en la fuerte represión a los opositores, apoyados en la implementación de una policía política, ACIC (KCIA por su sigla en inglés).

En el caso chileno, se impuso un régimen cívico-militar, hegemonizado por las FF.AA. y de orden, con un enfoque de "Estado desarrollista autoritario" en la década de 1970, visión expresada en la Declaración de Principios del Gobierno de Chile (1974), que derivó a un modelo de tipo neoliberal que combinó el autoritarismo político (sustentado en la Constitución de 1980) con el liberalismo económico. El liberalismo económico estuvo centrado en la apertura hacia el exterior, el aislamiento y blindaje de la tecnocracia que implementaba la nueva política económica y en la formación de coaliciones relativamente estables y duraderas entre los militares, los tecnócratas económicos y los empresarios (Silva, 2000). Como han planteado Huneeus (2000) y Valdivia (2003), se pasó por una etapa de ajuste interno en que las diferentes visiones de quienes apoyaban al gobierno de entonces debieron someterse al enfoque ortodoxo, como doctrina única.

En ambos casos se puede observar que el éxito económico estuvo mediado por el autoritarismo político y por los excesos de una represión hacia los opositores, cuestión que fue vista como el 
precio que debía pagarse por los resultados obtenidos (Fermandois, 2004), pero que, sin embargo, constituyó la clave de la pérdida de legitimidad y del poder. Como planteó Domínguez (2011), pese a que Chile y Corea del Sur estaban en la reducida lista de "dictaduras perfectas", ambas sucumbieron por las consecuencias de sus atropellos frente a las demandas por mayor democracia.

Ambos regímenes tuvieron proyectos refundacionales que implicaron el establecimiento de nuevas cartas constitucionales (Huneeus, 2000) (Dixon y Ginsburg, 2014) (Seo y Lim, 2015) (Choi, 2012): En Corea del Sur en 1972 y en 1980; y en Chile en 1980. En ambos casos, con el objeto de afianzar de manera permanente tanto a sus dictaduras como a sus líderes, lo que fue realizado mediante la purga de las judicaturas, previo al establecimiento de las constituciones, y la legalización del autoritarismo (Ginsburg, 2011), una vez promulgadas las nuevas Cartas Magnas. En ambos casos, los excesos se ampararon en estas Constituciones y, paradójicamente, en ambos, las rutas hacia la democracia estuvieron delineadas por estas leyes fundamentales que, en sus diseños, contemplaban que los líderes autoritarios ganasen las elecciones de manera ininterrumpida. En ambos casos, las elecciones demostraron lo opuesto.

\subsection{Paralelismo: Park Chung-hee $y$ Augusto Pinochet}

Usando la metáfora de Domínguez (2011), no se podría afirmar de que se trate de "perfectos dictadores", pero sí que ellos podrían ser concebidos, como sugieren Corner y Lim (2016) o De Mesquita y Smith (2011), como arquetipos, que actuaron en la misma época, sujetos a procesos históricos muy equivalentes $\mathrm{y}$, en consecuencia, comparables (Landman, 2011).

Estos dos líderes fueron militares de carrera, adscritos al Estado en una época de Estados sin contrapeso frente a la sociedad civil y al empresariado privado. Del mismo modo, coincidieron en no formar parte de las élites tradicionales de sus países. Ambos compartían una visión radicalmente antimarxista y pro-Occidente: el coreano más inclinados hacia Estados Unidos y el chileno más inclinado hacia Inglaterra (River, 2016).

Se trató de liderazgos susceptibles de formar alianzas por conveniencia y por principios. A ello se sumó, como muchas veces en la Historia, el azar: después del atentado que costó la vida a la segunda esposa de Park Chung-hee, Yuk Young-soo, perpetrado el 15 de agosto de 1974, el vínculo entre Park y Pinochet cambió y ambos jefes de Estado llevarían su relación y su confianza inicial a un nivel más alto.

De manera muy discreta, se nombró al yerno de Park, Byung Ki Han, como embajador de Corea del Sur en Chile, en una fecha aún indeterminada en las fuentes oficiales, pues hay un solo registro de su presencia en el país (AGHMRREEa, 1975). Su nombramiento no aparece ni en las memorias anuales del Ministerio de Relaciones Exteriores de Chile, ni en el Archivo General Histórico de la Cancillería chilena. Sí hay evidencia de su partida, en una nota del 13 de junio de 1975 (AGHMRREEd, 1975), lo que también quedó registrado por el Action ARA-Bureau of Inter-American Affairs del Departamento de Estado norteamericano, que se refiere a Han como alguien que "está obviamente muy bien conectado en Corea, aparentemente a través de su esposa" (1975), quien jamás apareció nombrada, a pesar de que el protocolo diplomático obliga a dejar registro de ese tipo de información. Esta misteriosa mujer era, Park Jae-ok, hija que Park Chung-hee había tenido en su primer matrimonio con Kim Ho-nam (GENI, 2019) y que habría sido sacada de Corea después del atentado sufrido por su padre en 1974.

Esta información, desconocida hasta el presente, le confiere otra valoración a las relaciones entre Chile y Corea del Sur de estos años, pues ya no se trató solo de una opción pragmática para mitigar el aislamiento internacional del gobierno cívico-militar de Chile o de incrementar el comercio internacional del país, sino que de una relación que podría haber alcanzado connotaciones mayores como la de una alianza estratégica política, especialmente entre dos líderes de dos regímenes que de suyo ya eran altamente convergentes.

A partir de estos gestos de confianza y lealtad, la alianza entre el régimen liderado por Park y encabezado por Pinochet, de algún modo, quedó sellada. De aquí en delante de construiría otra relación y otra historia.

\section{Conclusiones}

La etapa previa al momento que ocupa a este trabajo, si bien estuvo marcada por fenómenos importantes, no logró producir un efecto relevante en las relaciones sujetas a este estudio. 
Las relaciones políticas entre Chile y Corea del Sur, formalizadas a comienzos de la década de 1960, se estructuraron bajo las características propias de la Guerra Fría de entonces, lo que obligó a una definición de Chile en la cuestión de la unificación de la Península, a favor de Corea del Sur.

Pese a la relevancia de dicho asunto, la relación entre Chile y Corea del Sur era de baja intensidad. Como se ha planteado, solo después de 6 años de iniciadas formalmente se suscribió un convenio básico de cooperación económica y técnica, lo que no alteró el nivel de la interacción precedente.

En 1970, el triunfo de la Unidad Popular implicó un cambio importante en las relaciones de Chile con Corea, por cuanto se abrieron relaciones con los países de la órbita socialista y, entre ellos, con Corea del Norte, lo que tensionó la relación que se había iniciado una década antes con Corea del Sur. Sin embargo, salvo por un viaje del canciller chileno, Clodomiro Almeyda, que en gira por Asia visitó este país, el vínculo entre Chile y Corea del Norte no alcanzó a desarrollarse de manera importante antes del abrupto término del gobierno de Allende.

La relación bilateral que se configuró a partir del 11 de septiembre de 1973 entre Chile y Corea, tuvo un carácter estratégico, en tanto se relacionó con aspectos vitales para la supervivencia de los regímenes y sus principales integrantes.

Como se ha planteado en otros trabajos, en el ámbito internacional, la dictadura sobrevivió por una política exterior bastante más sofisticada que la representada por el llamado "estilo diplomático pretoriano ideológico", cuya acción tuvo especial éxito con los países de regímenes políticos del mismo signo y con países como los del Este y Nor-Este de Asia, que combinaban economías emergentes con visiones pragmáticas de la política internacional, transformándoles en socios perfectos para un país internacionalmente cuestionado, como fue el caso de Chile.

A esta conveniente neutralidad asiática, se agregó el efecto del golpe de Estado en Chile, frente al que el gobierno coreano respondió con el casi inmediato reconocimiento del nuevo gobierno (24 de septiembre de 1973). De allí hasta el mes de junio de 1975, cuando el embajador chileno concluyó las visitas protocolares, las relaciones bilaterales se elevaron de estatus, desde un punto de vista real y simbólico.

En este contexto, de por sí positivo para el gobierno chileno, se produjo el atentado al presidente Park, lo que desencadenó la muerte de su segunda esposa y la salida forzada de una de sus hijas rumbo a Chile. Esta pequeña historia, desconocida hasta el presente, cambia la perspectiva de interpretación de estas relaciones, en tanto no se trata solo de una opción pragmática para mitigar el aislamiento internacional del gobierno cívico-militar de Chile o de incrementar el comercio internacional del país, sino que de una relación estratégica para los países y para sus líderes.

A partir de estos gestos de confianza y lealtad, la alianza entre el régimen liderado por Pinochet y el encabezado por Park, de algún modo, quedó sellada. De aquí en delante de construiría otra relación y otra historia.

\section{Referencias Citadas}

Álvarez, R.

2017 La Estrategia Comunicacional de China hacia América del Sur. Santiago: Ediciones Universidad Finis Terrae.

Álvarez, R.

2018 China: entre el posicionamiento y la re-significación global, el rol de Xi Jinping. Revista Encrucijada Americana, 10 (1), 121-139.

$\mathrm{BCN}$.

2012 Dos Voluntades para el desarrollo. 50 años de relaciones entre Chile y Corea. Santiago: Biblioteca del Congreso Nacional.

Braudel, Fernand

2002 "La Historia y las Ciencias Sociales", Alianza Editorial, Madrid-España.
Choi, L.

2012 The Foreign Policy of Park Chunghee: 1968-1979 (Thesis degree of Doctor of Philosophy). London: Department of International History of The London School of Economics and Political Science.

Department of State.

28 de mayo de 1975 Public Library of US Diplomacy. Recuperado el octubre de 2018, de Wikileaks: https:// wikileaks.org/plusd/cables/1975SANTIA03531_b.html

Corner, P. y Lim, J.

2016 The Palgrave Handbook of Mass Dictatorship. London: Palgrave McMillan.

De Mesquita, B. y Smith, A.

2011 The Dictator's Handbook: Why Bad Behavior is Almost Always Good Politics,. New York: Public Affairs. 
Dixon, R. y Ginsburg, T.

2014 Comparative Constitutional Law in Asia. Northampton: Edward Elgar.

Domínguez, J.

2011 The Perfect Dictatorship? South Korea, versus Argentina, Brazil, Chile, and Mexico. En K. Byung-Kook, \& E. Vogel, The Park Chung-hee Era. The Transformation of South Korea (págs. 573-602). Cambridge: Harvard University Press.

Evans, G. y Newnham, J.

1998 The Penguin Dictionary of International Relations. London. United Kingdom: London: Penguin.

Fermandois, J.

1985 Chile y el Mundo 1970-1973. La Política Exterior del Gobierno de la Unidad Popular y el Sistema Internacional. Santiago: PUC.

Fermandois, J.

1991 De una inserción a otra: Política Exterior de Chile, 1966-1991. Revista de Estudios Internacionales, 24 (96), 433-455.

Fermandois, J.

2004 Mundo y Fin de Mundo: Chile en la Política Mundial 1900-2004. Santiago: PUC.

Fernández, Antoni Santisteban

2017 Del tiempo histórico a la conciencia histórica: cambios en la enseñanza y el aprendizaje de la historia en los últimos 25 años. Diálogo Andino [online], 2017, n.53 [citado 2020-09-27], pp. 87-99. Disponible en: <https:// scielo.conicyt.cl/scielo.php?script=sci_arttext\&pid=S0719$26812017000200087 \& \operatorname{lng}=\mathrm{es} \& n r m=i s o>$. ISSN $0719-2681$, http://dx.doi.org/10.4067/S0719-26812017000200087.

García-Blanch, F.

2002 Crecimiento económico en Corea del sur (1961-2000) Aspectos internos y factores internacionales. Madrid: Síntesis.

Ginsburg, T.

2011 Constitutional endurance. En T. Ginsburg, \& D. Rosalind, Research Handbooks in Comparative Law. Northampton: Edward Elgar Publishing Limited.

Greco, O.

2007 Diccionario de Política. Buenos Aires: Valletta Ediciones.

Hollis, Martin \& Smith, Steve Smith

1990 Explaining and Understanding International Relations, Oxford University Press, Oxford-UK.

Huguet Santos, Montserrat

2001 "Historia del Tiempo Presente e Historia de las Relaciones Internacionales, en Historia de las Relaciones Internacionales", AYER, No 42 (Parcial Pons), Madrid, España, pp. 43 y ss.

Huneeus, C.

2000 El Régimen de Pinochet. Santiago: Sudamericana.

Landman, T.

2011 Política Comparada. Una introducción a su objeto y métodos de investigación. Madrid: Alianza Editorial.

León, J.

2006 Autoritarismo y democracia en Corea del Sur: teoría y realidad. Los intersticios de la democracia y el autoritarismo. Algunos casos de Asia, África y América Latina. Buenos Aires: CLACSO.

McLean, I. y McMillan, A.

2009 The Concise Oxford Dictionary of Politics. Oxford: Oxford University Press.
Morgenthau, $\mathrm{H}$.

1948 Politics Among Nations. New York, USA: Alfred A. Knopf.

Morley, M. y McGillion, C.

2015 Reagan and Pinochet. The Struggle over U.S. Policy toward Chile. Cambridge: Cambridge University Press.

Muñoz, Heraldo

1986 Las Relaciones Exteriores del Gobierno Militar Chileno. Santiago: PROSPEL-CERC.

Palma, P. y Montt, M.

2017 La diáspora china en Iquique y su rol en la política de ultramar durante la República y el inicio de la Guerra Fría (1911-1950). Diálogo Andino [online], 2017, n. 54 [citado, 2020-09-27], pp. 143-152. Disponible en: <https:// scielo.conicyt.cl/scielo.php?script=sci_arttext\&pid=S0719$26812017000300143 \& \operatorname{lng}=e s \& n r m=i s o>$. ISSN 0719-2681, http://dx.doi.org/10.4067/S0719-26812017000300143.

River, $\mathrm{C}$.

2016 Augusto Pinochet: The Life and legacy of Chile's Controversial Dictator. Cambrige: Charles River Editors.

Robertson, D.

2007 The Routledge Dictionary of Politics. New York: Routledge.

Rodrik, D.

1993 Taking trade Policy Seriously: Export subsidization as a case Study in Policy effectivenes. Working Paper $\mathrm{N}^{\circ} 4567$.

Romero, A.

2005 El sistema político autoritario de Corea del Sur: Historia, desarrollo económico y democratización. Obtenido de Centro de Estudios Corea Argentina (CECA): http://www.uba.ar/ ceca/download/sistema-politico-de-corea-del-sur.pdf

Ross, C.

2007 Chile y Japón, 1973-1989: De la incertidumbre a la alianza estratégica. Santiago: LOM-USACH.

Ross, C.

2014 Chile y Japón durante los mil días de Allende, 19701973: El camino lateral. Universum, 29 (2), 277-290.

Ross, C.

27-28 de febrero de 2016. The coup in Chile in 1973 and the banality of Evil. Coup D'etat in the World and Turkey International. Estambul, Turquía.

Ross, C.

2018 Chile-Japón, 1990-2007: avances y repliegues en una alianza estratégica fallida. Diálogo Andino, № 56, Chile, (56), 101-117.

Ross, C. y Álvarez, R.

2018 Corea del Sur y América del Sur: Lecciones de dos trayectorias. Santiago: ChKSCP-IDEA.

Sater, W.; Morley, M. y McGillion, C.

2015 Reagan and Pinochet. The Struggle over U.S. Policy toward Chile. Cambridge:: Cambridge University Press.

Seo, J. y Lim, S.

2015 Civil Society under Authoritarian Rule: Bansanghoe and Extraordinary Everyday-ness in Korean Neighborhoods. Korea Journal, 55 (1), 59-85.

Sigmund, P.

1993 The United States and Democracy in Chile. Baltimore: The John Hopkins University Press.

Silva, P.

2000 State capacity, technocratic insulation, and governmentbusiness relations in South Korea and Chile. Santiago: FLACSO.

Smith, Peter et al.

2003 East Asia and Latin America. The Unlikely Alliance. New York: Rowman \& Littlefield Publishers. 
Spring, $\mathrm{M}$.

1980 Los países parias: realidad y potencial del quinto mundo. Santiago: Editorial Andrés Bello.

Spykman, N.

1942 America's strategy in world politics, the United States and the balance of power. New York: Harcourt, Brace \& Co.

Toro-Ibacache, L.

2018 La Política Exterior de Seguridad Japonesa: Historia y Teoría. Santiago: Ariadna.

Valdivia, V.

2003 El golpe después del golpe. Leigh vs. Pinochet. Chile (1960-1980). Santiago: LOM.

Westad, O. A.

2006 The Global Cold War: Third World Interventions and the Making of Our Times. Cambridge: Cambridge University Press.

Wolfsfeld, G.

2015 Political Actors. Revisado el 25 diciembre 2019 de: INFOCORE: (IN)Forming Conflict Prevention, Response and Resolution (INFOCORE) Definitions: www.infocore. eu/wp-content/uploads/2017/02/Def_Political_Actors.pdf

Ziring, L.

1995 International Relations: A Political Dictionary. Santa Barbara: ABC-CLIO.

\section{Fuentes}

\section{AGHMRREE.}

(24 de setiembre de 1973). Suk Heun Yun, Ministro de Relaciones Exteriores Subrogante de la República de Corea. Nota KCP-73-77. Seúl.

\section{AGHMRREEa.}

(24 de febrero de 1975). Remite Instrucciones al Embajador de parte del Subsecretario de Relaciones Internacionales, Coronel de Ejército, Enrique Valdés Puga, (DIRELAFRI). Oficio Reservado $N^{o} 1,1$. Santiago.

\section{AGHMRREEb.}

(21 de abril de 1975). Informa sobre instalación EMBACHILE SEÚL. Primeras diligencias. Impresiones. Oficio Ordinario $N^{o}$ 25/1. Seúl.

AGHMRREEc.

(21 de mayo de 1975). Instrucciones sobre uso de balija diplomática. Oficio Ordinario $N^{o} 41 / 2$. Seúl.

\section{AGHMRREEd.}

(13 de junio de 1975). Acusa recibo información sobre nuevo Embajador de Corea en Chile. Oficio Reservado $N^{o} 111 / 5$. Seúl.

\section{AGHMRREEe.}

(19 de junio de 1975). Informe sobre visitas protocolares. Oficio Reservado $N^{\circ} 118 / 7$. Seúl.

\section{AGHMRREEf.}

(5 de noviembre de 1975). Visita del Almirante José Toribio Merino. Oficio Reservado $N^{o} 372 / 85$. Santiago.

\section{Sitio web}

\section{GENI}

(8 de octubre de 2019). GENI. Obtenido de https://www. geni.com/people/Park-Chung-hee-3rd-President-of-theRepublic-of-Korea/6000000013355673919

\section{Notas}

1 Entendida como la expresión del pragmatismo asiático, que le llevó a prescindir de cualquier tipo de consideración de principios en su relación con Chile, centrando su vínculo solo en criterios asociados a sus intereses económicos. Específicamente, asegurar su provisión de recursos naturales para su industria manufacturera.

2 Entre estos: China, Japón, Hong Kong, Taiwán y Corea del Sur.

3 En el caso chino, este vínculo se había comenzado a desarrollar desde antes del establecimiento mismo de relaciones diplomáticas por medio, por ejemplo, de la migración (Palma y Montt, 2017).

4 Esta es una característica que ya constituía un patrón en la fase previa al período del gobierno chileno analizado en este trabajo (1973-1989) y que continúa más allá de su término (Ross, 2018).

5 He desarrollado una metodología de análisis de las visitas internacionales, más allá de las implicancias obvias que se puedan desprender de ellas (Ross, 2018).

$6 \quad$ Político y militar de Corea del Sur, educado en la Academia Militar de Corea y en la Universidad Nacional de Seúl. Fundador de la Agencia Central de Inteligencia Coreana (ACIC, actualmente el Servicio de Inteligencia Nacional de Corea del Sur) que sirvió como primer ministro dos veces, en los períodos de 1971-1975 y 1998-2000.

7 Casado con Park Yeong-ok
8 En la reunión con el jefe de los asuntos americanos del Ministerio de Relaciones Exteriores, el embajador chileno se vio consultado por la reunión sostenida entre el Jefe de Estado de Chile y su homónima argentina, María Martínez de Perón, por las tensiones entre ambos países. El embajador argumentó que el tema del Beagle estaba en vías de arreglo y que no afectaba las relaciones entre ambos. En diciembre de 1978, Argentina y Chile estuvieron a muy poco de entrar en guerra.

9 Entre otros, Argentina, Bolivia, Brasil, Chile, Paraguay y Uruguay, conformaron el Plan Cóndor, lo que les cohesionó más allá de sus coincidencias ideológicas.

10 Perú, tanto por su conflictividad histórica como por el enfoque ideológico del su gobierno. Italia, por la orientación política de su gobierno.

11 En su origen etimológico el concepto estrategia proviene de dos palabras en griego, "stratos" (ejército) y "agein" (guía o conductor) (McLean \& McMillan, 2009) (Greco, 2007). De aquí entonces que, para la mayoría de los autores, la estrategia esté asociada con los estudios militares y el de táctica, que se refiere a la forma (método) a emplear para lograr los objetivos planteados en una estrategia. En un sentido más amplio, se ha utilizado para referirse al plan complejo destinado a lograr una meta, de cualquier naturaleza -no solo militar-, de la manera más óptima posible. Cuando el grado de dificultad para alcanzar las 
metas es muy alto, los actores involucrados -estatales y no estatales- se abren a considerar la posibilidad de crear formas de asociación (Wolfsfeld, 2015). A su vez, cuando estas asociaciones tienen un alcance y un plazo mayor (Robertson, 2007), pueden denominarse asociaciones o alianzas estratégicas.
12 El gobierno cívico militar era tan aliado de la República Popular de China, como de Sudáfrica o Paraguay.

13 En cierto modo, adquirieron un carácter permanente en lo que podría denominarse la conciencia histórica de la política exterior de Chile (Fernández, 2017). 